\title{
Complete mitogenomes of Anopheles peditaeniatus and Anopheles nitidus and phylogenetic relationships within the genus Anopheles inferred from mitogenomes
}

\author{
Jing Guo, Zhen-Tian Yan, Wen-Bo Fu, Huan Yuan, Xu-Dong Li and Bin Chen* ${ }^{*}$
}

\begin{abstract}
Background: Despite the medical importance of mosquitoes of the genus Anopheles in the transmission of malaria and other human diseases, its phylogenetic relationships are not settled, and the characteristics of mitochondrial genome (mitogenome) are not thoroughly understood.

Methods: The present study sequenced and analyzed the complete mitogenomes of An. peditaeniatus and An. nitidus, investigated genome characteristics, and inferred the phylogenetic relationships of 76 Anopheles spp.

Results: The complete mitogenomes of An. peditaeniatus and An. nitidus are 15,416 and 15,418 bp long, respectively, and both include 13 PCGs, 22 tRNAs, two tRNAs and one control region (CR). Mitogenomes of Anopheles spp. are similar to those of other insects in general characteristics; however, the $\operatorname{trn} R$ and $\operatorname{trn} A$ have been reversed to "trnR-trnA," as has been reported in other mosquito genera. Genome variations mainly occur in CR length (493-886 bp) with six repeat unit types identified for the first time that demonstrate an evolutionary signal. The subgenera Lophopodomyia, Stethomyia, Kerteszia, Nyssorhynchus, Anopheles and Cellia are inferred to be monophyletic, and the phylogenetic analyses support a new phylogenetic relationship among the six subgenera investigated, in that subgenus Lophopodomyia is the sister to all other five subgenera, and the remaining five subgenera are divided into two clades, one of which is a sister-taxon subgenera Stethomyia + Kerteszia, and the other consists of subgenus Nyssorhynchus as the sister to a sister-group subgenera Anopheles + Cellia. Four series (Neomyzomyia, Pyretophorus, Neocellia and Myzomyia) of the subgenus Cellia, and two series (Arribalzagia and Myzorhynchus) of the subgenus Anopheles were found to be monophyletic, whereas three sections (Myzorhynchella, Argyritarsis and Albimanus) and their subdivisions of the subgenus Nyssorhynchus were polyphyletic or paraphyletic.
\end{abstract}

Conclusions: The study comprehensively uncovered the characteristics of mitogenome and the phylogenetics based on mitogenomes in the genus Anopheles, and provided information for further study on the mitogenomes, phylogenetics and taxonomic revision of the genus.

Keywords: Mitogenomes, Phylogenetics, Culicidae, Anopheles, Anopheles peditaeniatus, An. nitidus

*Correspondence: bin.chen@cqnu.edu.cn

Chongqing Key Laboratory of Vector Insects, Institute of Entomology and Molecular Biology, College of Life Sciences, Chongqing Normal

University, Chongqing 401331, People's Republic of China

\section{Background}

The genus Anopheles belongs to the subfamily Anophelinae in Culicidae (mosquitoes). It is the most diverse genus in the subfamily, with 475 formally named species and more than 50 unnamed members of species original author(s) and the source, provide a link to the Creative Commons licence, and indicate if changes were made. The images or other third party material in this article are included in the article's Creative Commons licence, unless indicated otherwise in a credit line to the material. If material is not included in the article's Creative Commons licence and your intended use is not permitted by statutory regulation or exceeds the permitted use, you will need to obtain permission directly from the copyright holder. To view a copy of this licence, visit http://creativecommons.org/licenses/by/4.0/. The Creative Commons Public Domain Dedication waiver (http://creativeco mmons.org/publicdomain/zero/1.0/) applies to the data made available in this article, unless otherwise stated in a credit line to the data. 
complexes worldwide [1]. Anophelinae mosquitoes can transmit a variety of diseases, and are one of the most important groups of insects in medicine, as they are the unique vectors of human malarial parasites, which caused 229 million cases and 409,000 deaths worldwide in 2019 [2]. In addition to malaria parasites, mosquitoes in Anopheles also transmit filarial parasites [3]. Some studies have shown that Anopheles mosquitoes also harbor arboviruses, which multiply in the mosquito vectors before transmission to a vertebrate host, such as o'nyongnyong [4]. Due to their exceeding importance, mosquitoes of this genus are subject to more taxonomic studies than any other mosquito group.

The classification of Anopheles started more than 100 years ago [5], when it was treated as one of 18 genera in the Anophelinae, while Cellia, Nyssorhynchus, Stethomyia and Kerteszia were also treated as independent genera based on morphological characteristics. Subsequently, the five genera were successively included as subgenera of the genus Anopheles based on the number and location of specialized setae on the male genital gonocoxites and other characteristics [6-8]. Three additional subgenera, Lophopodomyia, Baimaia and Christya were established within the genus Anopheles [9-11]. Due to the diversity of species contained in the subgenera Anopheles, Cellia and Nyssorhynchus, taxonomists divided some species into informal categories such as sections, series and groups. The earliest phylogenetic studies for Anopheles were mainly based on morphological characters and single genes. Different data sets and phylogenetic inference methods often lead to inconsistent results between studies, and therefore phylogenetic relationships in Anopheles have not been well settled.

There have been a number of representative phylogenetic studies on the genus Anopheles. An analysis including 63 species in Anophelinae based on 163 morphological characters suggested the monophyly of the subgenera Cellia, Nyssorhynchus, Stethomyia, Kerteszia and Lophopodomyia [12]. In Nyssorhynchus, the three sections Albimanus, Argyritarsis and Myzorhynchella were suggested to be paraphyletic. In Cellia, only the series Cellia was considered to be monophyletic. In Anopheles, series Arribalzagia and Lophoscelomyia were considered to be monophyletic, while the series Cycloleppteron + Arribalzagia was nested within series Myzorhynchus [12]. Some further morphology-based studies also suggested the monophyly of the subgenera Nyssorhynchus, Cellia and Kerteszia, and displayed the sister relationship between subgenera Kerteszia and Nyssorhynchus [11, 13, 14]. An analysis based on COX1 + ITS2 dataset suggested the monophyly of subgenera Anopheles and Cellia, and the analysis using ITS2 dataset alone resulted in the same conclusion, which was not supported by the COX1 dataset alone [15]. Two studies based on the mitogenomes, including 50 and 33 species, respectively, both also supported the monophyly of the subgenera Anopheles, Nyssorhynchus, Cellia and Kerteszia [16, 17]. Generally, the monophyly of the subgenera Anopheles, Nyssorhynchus, Cellia, Stethomyia, Kerteszia and Lophopodomyia has been supported by most recent studies; however, sections and series within the subgenera Anopheles, Nyssorhynchus and Cellia have not been well resorted. There is a need to elucidate the phylogeny of the genus Anopheles using more species, more data and updated phylogenetic analysis approaches.

The mitochondrion is an important organelle in eukaryotic cells, with a genome independent of the nucleus, the mitochondrial genome (mitogenome) [18]. The mitogenome typically has a small genome size, low levels of recombination and maternal inheritance, and therefore it has been widely used as a molecular marker for the identification of species, phylogenetic inference and population structure research $[19,20]$. Since the publication of the first insect mitogenome (Drosophila yakuba) in 1985 [21], the number of insect mitogenomes have increased rapidly. Phylogenetic studies based on insect mitogenomes have shown good results in Diptera [22], Orthoptera [23], Coleoptera [24] and Hymenoptera [25]. To date the complete mitogenomes of 125 species of Culicidae have been sequenced, of which 74 species are from the genus Anopheles. Dipteran mitogenomes are mostly $14-20 \mathrm{~kb}$ long, including 37 genes -13 proteincoding genes (PCGs), two ribosomal RNA (rRNA) genes, 22 transfer RNA (tRNA) genes and a control region $(\mathrm{CR})$ - and these genes are arranged in a compact circular genome [26]. The genome structure in all reported mosquito mitogenomes is similar to the typical mitogenomes of Diptera; however, the $\operatorname{trn} A$ and $\operatorname{trn} R$ of mosquitoes are rearranged to form "trnR-trnA" [16, 17, 21].

In the present study, we sequenced and annotated the complete mitogenomes of An. peditaeniatus and An. nitidus, and analyzed the mitogenome characteristics of 76 species in the genus Anopheles. Additionally, we constructed the phylogenetic relationships of these 76 species. This study provides new insights into the mitogenome characteristics and phylogenetic relationships in the genus Anopheles.

\section{Methods}

\section{Sample collection and DNA extraction}

Specimens of An. peditaeniatus and An. nitidus were collected from Yadong County $\left(29^{\circ} 11^{\prime} 46^{\prime \prime} \mathrm{N}, 95^{\circ} 12^{\prime} 11^{\prime \prime} \mathrm{E}\right)$, Tibet, China, in July 2014, and Tiebei County, Jilin Province, China ( $\left.42^{\circ} 27^{\prime} 21^{\prime \prime} \mathrm{N}, 128^{\circ} 06^{\prime} 18^{\prime \prime} \mathrm{E}\right)$ in July 2013. All samples were preserved in individual vials. After morphological identification using keys reported previously 
[27], samples were stored in $100 \%$ alcohol and housed at $-20{ }^{\circ} \mathrm{C}$ until DNA extraction. Total DNA was extracted from an individual adult mosquito using the QIAGEN Genomic DNA Kit [28], and used for 350 bp library construction and Illumina high-throughput sequencing by Shenzhen Huitong Biotechnology Co. Ltd.

\section{Mitogenome sequencing annotation and characteristics analysis}

Genome sequencing using paired-end sequencing (PE 150) was carried out using the Illumina HiSeq $X$ Ten platform by Huitong Biotechnology Co., Ltd. In total, $20.41 \mathrm{~Gb}$ (An. peditaeniatus) and $25.96 \mathrm{~Gb}$ (An. nitidus) clean data were obtained after filtering of raw data (20.54 Gb for An. peditaeniatus and $26.15 \mathrm{~Gb}$ for $A n$. nitidus) using the NGS QC Toolkit [29], and the sequencing depth was 288.9X (An. peditaeniatus) and 5162X (An. nitidus). Subsequently, the mitogenome reads were extracted using the BLAST program with An. sinensis mitogenome sequence as reference, and assembled using de novo mitogenome assembly with SPAdes 3.9.0 [30].

The mitogenomes of $A n$. peditaeniatus and An. nitidus were annotated using MITOS (http://mitos.bioinf. unileipzig.de/index.py) [31]. Protein-coding gene and ribosomal RNA gene annotations were confirmed by reference to published mosquito mitogenomes and corrected in Geneious v4.8.5 [32]. The secondary structures of tRNAs were predicted using MITOS [31], and the structure maps of the mitogenomes were visualized using OGDRAW1.3.1 [33]. Base composition, codon usage, relative synonymous codon usage (RSCU) and amino acid content were computed with MEGA v.7.0.26 [34]. Nucleotide compositional bias was calculated using the formulas $\mathrm{AT}$-skew $=[\mathrm{A}-\mathrm{T}] /[\mathrm{A}+\mathrm{T}]$ and $\mathrm{GC}$-skew $=[\mathrm{G}-\mathrm{C}] /$ $[\mathrm{G}+\mathrm{C}]$ [35], and three-dimensional scatter plots of AT-skew, GC-skew and AT\% were drawn using OriginPro v.9.0 [36]. Selection pressure on the 13 PCGs was analyzed by calculating $\mathrm{Ka}$ and $\mathrm{Ks}$ values using DnaSP v6.12.03 [37]. Sequence motifs in the CR were identified using Tandem Repeats Finder [38].

\section{Phylogenetic analysis}

Multiple sequence alignments of the PCGs were performed on the TranslatorX server (http://translatorx. co.uk/) using the MAFFT amino acid alignment mode. Gblocks with the default setting in TranslatorX was used to remove the ambiguously aligned positions. Individual alignments were concatenated in SequenceMatrix [39]. PartitionFinder 2.0 was used to determine the best-fit substitution model for each gene according to the Akaike information criterion (AIC), and the default values for the initial partition settings were applied [40]. Phylogenetic analyses were performed using maximum likelihood (ML) inference in IQ-TREE 1.6.10 [41] and Bayesian inference (BI) analysis in MrBayes v.3.2.7a [42] using Culex pipiens pallens as outgroup (Table 1). Bootstrap values were calculated using 1000 replicates for ML. BI was performed as two independent runs, each with four chains, and these chains ran simultaneously for 10,000,000 generations, with sampling every 1000 steps, and a $25 \%$ burn-in rate. Phylogenetic trees were drawn using FigTree v.1.4.4 (http://tree.bio.ed.ac.uk/software/ figtree/).

\section{Results \\ Nucleotide composition and genome organization}

The complete mitogenomes of $A n$. peditaeniatus (GenBank: MT822295) and An. nitidus (GenBank: MW401801) are both circular genomes with full lengths of 15,416 and $15,418 \mathrm{bp}$, respectively (Fig. 1). Both are composed of 37 genes (including 13 PCGs, 22 tRNA genes and two rRNA genes) and one control region (CR). There are 22 genes (nine PCGs and 13 tRNAs) located on the majority coding strand (J-strand), while the other 15 genes (four PCGs, nine tRNAs and two rRNAs) on the minority strand (N-strand). Compared with the typical Diptera mitogenome (e.g., Drosophila yakuba), both An. peditaeniatus and An. nitidus have a "trnR-trnA" rearrangement. The AT content of the mitogenomes of the two species is high, $78.32 \%$ and $78.26 \%$, respectively, with obvious AT bias (Additional file 1: Table S1). The AT-skew of An. peditaeniatus (0.0322) is higher than the average AT-skew of mosquito mitogenomes (0.0283), whereas the AT-skew of An. nitidus mitogenome (0.0266) is lower than the mosquito average. GC-skew in $A n$. peditaeniatus (-0.1587) and An. nitidus (-0.1536) was higher than the average $\mathrm{GC}$-skew value in mosquitoes investigated $(-0.16048)$.

The three-dimensional scatter plot of AT content, ATskew and GC-skew of mitogenomes in the genus Anopheles is shown in Fig. 2. AT-skew ranged from 0.005 in $A n$. gilesi to 0.043 in An. christyi. All mitogenomes display negative GC-skews ranging from -0.207 in $A n$. parvus to -0.136 in An. punctulatus. Most species of the subgenera Nyssorhynchus and Cellia have similar AT content and AT/GC-skew (closely distributed in the three-dimensional scatter plot), whereas species in the subgenera Lophopodomyia, Stethomyia, Kerteszia and Anopheles ae widely distributed in the plot for AT content, AT-skew and GC-skew.

\section{Protein-coding genes}

The total nucleotide lengths of the PCGs of An. peditaeniatus and An. nitidus was 11,223 and $11,168 \mathrm{bp}$, respectively. In $A n$. peditaeniatus, ATN is used as the start codon for all genes except $C O X 1$ and ND5, which use 
Table 1 Detailed sequence information of mitochondrial genomes used in the present phylogenetic analysis

\begin{tabular}{|c|c|c|c|c|c|c|c|}
\hline Sections/series & Species & Total size (bp) & PCG size (bp) & tRNA size (bp) & rRNA size (bp) & CR size (bp) & GenBank \\
\hline \multicolumn{8}{|l|}{ Subgenus Cellia } \\
\hline \multirow[t]{5}{*}{ Myzomyia } & An. aconitus & 15,359 & 11,224 & 1472 & 2114 & 519 & NC039540 \\
\hline & An. culicifacies & 15,364 & 11,194 & 1474 & 2121 & 535 & NC028216 \\
\hline & An. culicifacies B & 15,330 & 11,230 & 1474 & 2114 & 498 & NC027502 \\
\hline & An. funestus & 15,356 & 11,231 & 1477 & 2121 & 519 & NC038158 \\
\hline & An. minimus & 15,411 & 11,194 & 1476 & 2117 & 546 & NC028221 \\
\hline \multirow[t]{3}{*}{ Neocellia } & An. maculatus & 14,850 & 11,188 & 1479 & 2108 & N/A & NC028218 \\
\hline & An. splendidus & 15,362 & 11,224 & 1477 & 2121 & 510 & NC039397 \\
\hline & An. stephensi & 15,387 & 11,190 & 1477 & 2117 & 551 & NC028223 \\
\hline \multirow[t]{5}{*}{ Neomyzomyia } & An.cracens & 15,412 & 11,224 & 1482 & 2123 & 576 & NC020768 \\
\hline & An. dirus & 15,406 & 11,224 & 1478 & 2124 & 568 & NC036263 \\
\hline & An. farauti 4 & 15,412 & 11,224 & 1482 & 2125 & 576 & NC020770 \\
\hline & An. hinesorum & 15,336 & 11,224 & 1479 & 2123 & 505 & NC020769 \\
\hline & An. punctulatus & 15,322 & 11,187 & 1477 & 2118 & 493 & NC028222 \\
\hline \multirow{7}{*}{ Pyretophorus } & An. arabiensis & 15,369 & 11,194 & 1477 & 2122 & 530 & NC028212 \\
\hline & An. christyi & 14,967 & 11,188 & 1477 & 2126 & $\mathrm{~N} / \mathrm{A}$ & NC028214 \\
\hline & An. coluzzii & 15,441 & 11,194 & 1478 & 2124 & 599 & NC028215 \\
\hline & An.epiroticus & 15,379 & 11,188 & 1479 & 2122 & 535 & NC028217 \\
\hline & An.gambiae & 15,363 & 11,230 & 1479 & 2125 & 519 & NC002084 \\
\hline & An.melas & 15,366 & 11,194 & 1477 & 2122 & 526 & NC028219 \\
\hline & An.merus & 15,365 & 11,188 & 1478 & 2121 & 525 & NC028220 \\
\hline \multicolumn{8}{|l|}{ Subgenus Anopheles } \\
\hline \multirow[t]{4}{*}{ Angusticorn/Anopheles } & An. atroparvus & 15,458 & 11,175 & 1474 & 2161 & 614 & NC028213 \\
\hline & An. eiseni geometricus & 15,696 & 11,241 & 1474 & 2120 & 860 & MF381678 \\
\hline & An. lindesayi & 15,366 & 11,225 & 1475 & 2123 & 531 & KX961140 \\
\hline & An. quadrimaculatus A & 15,455 & 11,220 & 1473 & 2115 & 625 & NC000875 \\
\hline \multirow[t]{7}{*}{ Laticorn/Arribalzagia } & An. costai & 15,433 & 11,241 & 1473 & 2122 & 598 & NC037794 \\
\hline & An. nr. costai & 15,434 & 11,241 & 1473 & 2121 & 600 & NC037821 \\
\hline & An. fluminensis & 15,429 & 11,241 & 1474 & 2120 & 594 & NC037818 \\
\hline & An. forattinii & 15,459 & 11,241 & 1473 & 2125 & 615 & NC037813 \\
\hline & An. Medialis ${ }^{\text {a }}$ & 15,409 & 11,241 & 1475 & 2121 & 545 & NC037789 \\
\hline & An.minor & 15,466 & 11,238 & 1478 & 2123 & 594 & NC037802 \\
\hline & An. peryassui & 15,417 & 11,241 & 1474 & 2120 & 585 & NC037790 \\
\hline \multirow[t]{4}{*}{ Laticorn/Myzorhynchus } & An. coustani & 15,408 & 11,194 & 1475 & 2112 & 570 & MT806097 \\
\hline & An. nitidus & 15,418 & 11,168 & 1476 & 2122 & 580 & MW401801 \\
\hline & An. peditaeniatus & 15,416 & 11,224 & 1477 & 2125 & 575 & MT822295 \\
\hline & An. sinensis & 15,418 & 11,224 & 1473 & 2125 & 577 & MF322628 \\
\hline
\end{tabular}

TCG and GTG as start codons. In An. nitidus, all PCGs initiate with ATN as the start codon, except COX1, which uses TCG (Table 2).

The RSCU values of mitogenomes in the genus Anopheles are presented in Additional file 2: Table S2. Anopheles species have different usage frequencies of synonymous codons; UUA is the most frequently used codon, followed by CGA, GGA, GCU. The amino acid Leu has the highest usage percentage for all 76 mitogenomes investigated with an average of $16.37 \%$, followed by Phe (9.69\%), Ile (9.31\%) and Ser (8.48\%), whereas Cys has the lowest percentage $(0.99 \%)$. The usage percentages of amino acids do not differ significantly between different subgenera (Fig. 3).

The non-synonymous (Ka) and synonymous (Ks) substitution ratio $(\mathrm{Ka} / \mathrm{Ks})$ of the PCGs are shown in Fig. 4. The $\mathrm{Ka} / \mathrm{Ks}$ ratios are all less than 1 , with $N D 6$ the highest (0.203), followed by six genes (ATP8, ND2, ND5, ND4L, $N D 4, N D 3)$ with $\mathrm{Ka} / \mathrm{Ks}$ ratios of $0.098-0.152$. Complex IV (COX1, COX2 and COX3), Complex III (CYTB), ND1 and ATP6 have low $\mathrm{Ka} / \mathrm{Ks}$ ratios with range from 0.022 (COX1) to 0.051 (ND1). These results imply that all PCGs have experienced purifying selection, especially Complex IV, Complex III, ND1 and ATP6. 
Table 1 (continued)

\begin{tabular}{|c|c|c|c|c|c|c|c|}
\hline Sections/series & Species & Total size (bp) & PCG size (bp) & tRNA size (bp) & rRNA size (bp) & CR size (bp) & GenBank \\
\hline \multicolumn{8}{|l|}{ Subgenus Nyssorhynchus } \\
\hline \multirow[t]{14}{*}{ Albimanus/Oswaldoi } & An. albertoi & 15,385 & 11,240 & 1475 & 2114 & 558 & NC037804 \\
\hline & An. arthuri & 15,387 & 11,240 & 1475 & 2114 & 560 & NC037806 \\
\hline & An. benarrochi & 15,387 & 11,240 & 1477 & 2116 & 556 & NC037787 \\
\hline & An. evansae & 15,382 & 11,240 & 1477 & 2115 & 553 & NC037795 \\
\hline & An. galvaoi & 15,420 & 11,240 & 1477 & 2150 & 555 & NC037814 \\
\hline & An. goeldii & 15,391 & 11,240 & 1477 & 2117 & 560 & NC037810 \\
\hline & An. konderi & 15,395 & 11,240 & 1478 & 2125 & 555 & MF381685 \\
\hline & An. nuneztovari & 15,393 & 11,240 & 1477 & 2117 & 562 & MF381680 \\
\hline & An. oswaldoi & 15,380 & 11,237 & 1477 & 2115 & 554 & NC037793 \\
\hline & An. rangeli & 15,386 & 11,240 & 1477 & 2114 & 558 & NC037786 \\
\hline & An. rondoni & 15,385 & 11,240 & 1477 & 2113 & 557 & NC037815 \\
\hline & An. striatus & 15,385 & 11,240 & 1476 & 2115 & 557 & NC037801 \\
\hline & An. strodei & 15,388 & 11,240 & 1475 & 2115 & 560 & NC037808 \\
\hline & An. triannulatus & 15,401 & 11,240 & 1477 & 2125 & 559 & NC037800 \\
\hline \multirow[t]{9}{*}{ Argyritarsis/Albitarsis } & An. albitarsis & 15,413 & 11,216 & 1477 & 2119 & 575 & NC020662 \\
\hline & An. albitarsis F & 15,418 & 11,216 & 1479 & 2121 & 578 & NC030768 \\
\hline & An. albitarsis $\mathrm{G}$ & 15,474 & 11,216 & 1480 & 2125 & 615 & NC030766 \\
\hline & An. braziliensis & 15,397 & 11,240 & 1480 & 2115 & 562 & NC037791 \\
\hline & An. nr. braziliensis & 15,413 & 11,240 & 1478 & 2116 & 578 & MF381606 \\
\hline & An. deaneorum & 15,424 & 11,216 & 1476 & 2121 & 581 & NC020663 \\
\hline & An.janconnae & 15,425 & 11,216 & 1480 & 2120 & 575 & NC030767 \\
\hline & An. marajoara & 15,453 & 11,240 & 1476 & 2132 & 584 & NC037788 \\
\hline & An. oryzalimnetes & 15,422 & 11,216 & 1479 & 2120 & 581 & NC030765 \\
\hline \multirow[t]{5}{*}{ Argyritarsis/Argyritarsis } & An. argyritarsis & 15,403 & 11,240 & 1481 & 2115 & 579 & NC037807 \\
\hline & An. atacamensis & 15,412 & 11,241 & 1476 & 2122 & 564 & NC037792 \\
\hline & An. darlingi & 15,386 & 11,240 & 1489 & 2122 & 554 & NC014275 \\
\hline & An. lanei & 15,396 & 11,240 & 1478 & 2116 & 567 & NC037799 \\
\hline & An. sawyeri & 15,417 & 11,240 & 1477 & 2116 & 599 & NC037798 \\
\hline \multirow[t]{5}{*}{ Myzorhynchella } & An. antunesi & 15,427 & 11,242 & 1475 & 2118 & 595 & NC037817 \\
\hline & An. guarani & 15,531 & 11,241 & 1473 & 2119 & 700 & NC037816 \\
\hline & An. lutzii & 15,341 & 11,242 & 1475 & 2118 & 509 & NC037820 \\
\hline & An.parvus & 15,444 & 11,235 & 1470 & 2116 & 617 & NC037805 \\
\hline & An. pristinus & 15,405 & 11,241 & 1476 & 2117 & 581 & NC037824 \\
\hline \multicolumn{8}{|l|}{ Subgenus Kerteszia } \\
\hline & An. bellator & 15,668 & 11,242 & 1477 & 2126 & 811 & NC030249 \\
\hline & An. cruzii & 15,449 & 11,230 & 1478 & 2116 & 600 & NC024740 \\
\hline & An. homunculus & 15,739 & 11,242 & 1475 & 2125 & 886 & NC030248 \\
\hline & An. laneanus & 15,446 & 11,242 & 1479 & 2124 & 591 & NC030250 \\
\hline \multicolumn{8}{|l|}{ Subgenus Stethomyia } \\
\hline & An. kompi & 15,505 & 11,240 & 1476 & 2118 & 647 & NC037827 \\
\hline & An. nimbus & 15,476 & 11,240 & 1467 & 2121 & 628 & NC037811 \\
\hline \multicolumn{8}{|l|}{ Subgenus Lophopodomyia } \\
\hline & An. gilesi & 15,458 & 11,244 & 1465 & 2108 & 648 & NC037803 \\
\hline & An.pseudotibiamaculatus & 15,597 & 11,242 & 1478 & 2122 & 768 & NC037829 \\
\hline \multicolumn{8}{|l|}{ Outgroup } \\
\hline & Cx.pipiens pallens & 15,617 & 11,228 & 1482 & 2138 & 713 & KT851543 \\
\hline
\end{tabular}

\footnotetext{
${ }^{\mathrm{a}}$ Anopheles medialis $=$ Anopheles intermedius
} 

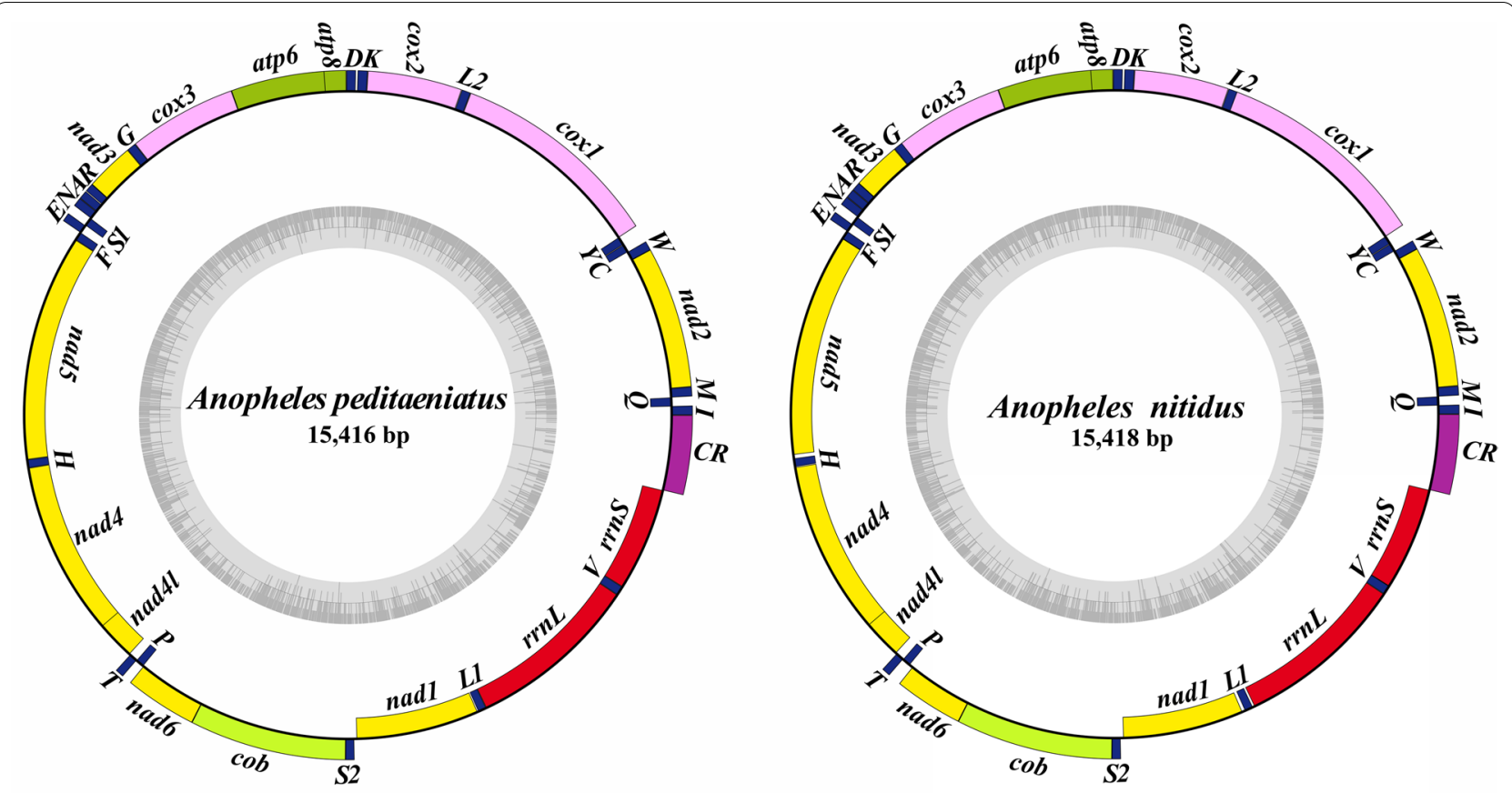

$\square$ complex I (NADH dehydrogenase)

$\square$ complex III (ubichinol cytochrome c reductase)

$\square$ complex IV (cytochrome c oxidase)

$\square$ ATP synthase

$\square$ other genes

$\square$ transfer RNAs

$\square$ ribosomal RNAs

Fig. 1 Mitochondrial genome structure of Anopheles peditaeniatus and Anopheles nitidus

\section{Transfer RNAs, ribosomal RNAs and CR}

The total length of tRNAs in An. peditaeniatus and $A n$. nitidus was $1475 \mathrm{bp}$ and $1476 \mathrm{bp}$, respectively, while the length of individual tRNAs varies from 64 to $72 \mathrm{bp}$. All tRNAs can fold into the typical clover-leaf structure of four stems and loops, except for trnS2 which has lost the dihydrouridine (DHU) arm (Additional file 3: Figure S1). The length of the rRNAs was $2125 \mathrm{bp}$, with an AT content of $81.36 \%$ in An. punctulatus and 2122 bp, with an AT content of $81.39 \%$ in An. nitidus.

The control regions (CRs) of Anopheles mitogenomes are located between $r r n S$ and $t r n I$, with lengths of 575 and 580 bp and AT content of $94.43 \%$ and $93.62 \%$ in $A n$. peditaeniatus and $A n$. nitidus, respectively. Six repeat unit types are found in the CRs of Anopheles mitogenomes (Additional file 4: Figure S2). All species have 15-27 bp poly-T stretch, located immediately after $140-$ $212 \mathrm{bp}$ of conserved sequence. The poly-T stretch is adjacent to the conserved motif $5^{\prime}$-CCCCTA- $3^{\prime}$ in 68 species, whereas this motif was replaced by $5^{\prime}$-ATTGTA- $3^{\prime}$ in $A n$. cracens and An. dirus, and 5'-TTCCCC- $3^{\prime}$ in An. kompi, An. nimbus, An. gilesi and An. pseudotibiamaculatus. The repeat type is $12-55$ bp long and composed of 2-6 repeats, located downstream of the poly- $T$ stretch, and is found in 54 species. The third type ([TA(A)] n stretch) with 22-91 repeats, is found in 36 species. The fourth type is a $12-38$ bp region composed of 2-5 repeats adjacent to trnI and found in 40 species. The remaining two repeat unit types are found in only a few species; one is a 15-36 bp region located after the second repeat type and found in five species, while the last type is a 108-171 bp region, the longest of the six types and found in only four species.

\section{Phylogenetic relationships}

Bayesian inference (BI) and maximum-likelihood (ML) analyses produced the same phylogenetic trees at the subgenus level (Figs. 5 and 6). The six subgenera investigated, Lophopodomyia, Stethomyia, Kerteszia, Nyssorhynchus, Anopheles and Cellia, are monophyletic in both analyses, with the posterior probability $(\mathrm{pp})=1$ for every subgenus (Fig. 5) and bootstrap values (bv) that range from 99 to $100 \%$ in ML analysis (Fig. 6). The subgenus Lophopodomyia is sister to remaining five subgenera, the clade of which has support of $\mathrm{pp}=0.99$ and bv $=71 \%$. The two subgenera Stethomyia and Kerteszia 




Fig. 2 Three-dimensional scatter plot of the AT-skew, GC-skew and AT\% of 76 mitochondrial genome sequences of the genus Anopheles

are sisters ( $\mathrm{pp}=1$ and $\mathrm{bv}=89 \%)$. The clade of Nyssorhynchus, Anopheles and Cellia was well supported ( $\mathrm{pp}=1$ and $\mathrm{bv}=68 \%$ ). The subgenus Nyssorhynchus is sister to the clade Anopheles + Cellia ( $\mathrm{pp}=1$ and $\mathrm{bv}=99 \%$ ).

In the subgenus Cellia, four series investigated, Myzomyia, Neocellia, Pyretophorus and Neomyzomyia, were each monophyletic $(\mathrm{pp}=1$ and $\mathrm{bv}=100 \%)$. The series Neomyzomyia was sister to the remaining three series. In the subgenus Anopheles, both Angusticorn and Laticorn were polyphyletic, while within section Laticorn both series Arribalzagia ( $\mathrm{pp}=1$ and $\mathrm{bv}=96 \%)$ and Myzorhynchus ( $\mathrm{pp}=1$ and $\mathrm{bv}=100 \%)$ were monophyletic. In Nyssorhynchus, all three sections investigated, Myzorhynchella, Argyritarsis and Albimanus, were polyphyletic, while in section Argyritarsis, both series Argyritarsis and Albitarsis were polyphyletic as well.

\section{Discussion}

Characteristics of the mitogenome sequences of the genus Anopheles

Comparison of mitogenome sequences in the genus Anopheles shows that the length variation mainly exists in the CRs, similar to earlier reported mitogenomes in insects $[43,44]$. The gene number and the gene composition, codon usage and tRNA secondary structures are similar to other reported mitogenomes of Diptera [22,
45]. However, the $\operatorname{trn} R$ and $\operatorname{trn} A$ have a reversed arrangement to form "trnR-trnA" in comparison to the ancestral insect, as those reported in other genera in Culicidae [21, 45].

The present study identified six repeat unit types in CRs for the first time in Anopheles mitogenomes. Among the six types, the poly- $T$ stretch has also been found in other insects, which may involve the identification of the replication origin of mitochondrial DNA (mtDNA) [46]. The conserved sequences in CRs have been reported to be taxon-specific and of evolutionary information, and have been used as important evidence in the inference of phylogenetics in the taxa of the genus Culex and Lutzia and taxon [47]. However, the evolutionary information carried in the genus Anopheles does not seem stable and reliable.

\section{Phylogenetic relationships}

This present study suggests that all six subgenera investigated are monophyletic, and the phylogenetic analysis shows that subgenus Lophopodomyia is the sister to all five other subgenera, and the remaining five subgenera are divided into two clades, one including a sister-taxon (Stethomyia + Kerteszia), and the other consisting of subgenus Nyssorhynchus as the sister to a sister-group subgenera Anopheles+Cellia. A phylogenetic study 
Table 2 Organization of the An. peditaeniatus and An. nitidus mitochondrial genomes

\begin{tabular}{|c|c|c|c|c|c|c|c|c|c|}
\hline \multirow[t]{2}{*}{ Gene } & \multirow[t]{2}{*}{ Strand } & \multicolumn{2}{|l|}{ Position (bp) } & \multicolumn{2}{|l|}{ Length (bp) } & \multicolumn{2}{|c|}{ Space(+)/overlap(-) } & \multicolumn{2}{|c|}{ Start/Stop codon } \\
\hline & & punctulatus & nitidus & punctulatus & nitidus & punctulatus & nitidus & punctulatus & nitidus \\
\hline $\operatorname{trnl}$ & J & $1-68$ & $1-68$ & 68 & 68 & 0 & 0 & & \\
\hline $\operatorname{trn} Q$ & $\mathrm{~N}$ & $66-134$ & $66-134$ & 69 & 69 & -3 & -3 & & \\
\hline $\operatorname{trnM}$ & J & 1134-202 & 134-202 & 69 & 69 & -1 & -1 & & \\
\hline nad2 & J & $203-1228$ & $203-1228$ & 1026 & 1026 & 0 & 0 & ATT/TAA & ATT/TAA \\
\hline $\operatorname{trn} W$ & J & $1227-1295$ & $1227-1295$ & 69 & 69 & -2 & -2 & & \\
\hline $\operatorname{trn} C$ & $\mathrm{~N}$ & $1295-1358$ & $1295-1358$ & 64 & 64 & -1 & -1 & & \\
\hline $\operatorname{trn} Y$ & $\mathrm{~N}$ & $1360-1425$ & $1360-1425$ & 66 & 66 & 1 & 1 & & \\
\hline $\operatorname{cox} 1$ & J & $1424-2960$ & $1424-2965$ & 1537 & 1542 & -2 & -2 & $\mathrm{TCG} / \mathrm{T}$ & TCG/TAA \\
\hline $\operatorname{trn} L 2$ & J & $2961-3026$ & $2961-3026$ & 66 & 66 & 0 & -5 & & \\
\hline $\cos 2$ & J & $3028-3712$ & $3028-3712$ & 685 & 685 & 1 & 1 & $\mathrm{ATG} / \mathrm{T}$ & $\mathrm{ATG} / \mathrm{T}$ \\
\hline $\operatorname{trnK}$ & J & $3713-3784$ & $3713-3784$ & 72 & 72 & 0 & 0 & & \\
\hline $\operatorname{trn} D$ & J & 3797-3865 & $3797-3865$ & 69 & 69 & 12 & 12 & & \\
\hline atp8 & J & $3866-4027$ & $3866-4027$ & 162 & 162 & 0 & 0 & ATT/TAA & ATT/TAA \\
\hline atp6 & J & $4021-4701$ & $4021-4701$ & 681 & 681 & -7 & -7 & ATG/TAA & ATG/TAA \\
\hline $\cos 3$ & J & $4701-5487$ & $4701-5495$ & 787 & 795 & -1 & -1 & $\mathrm{ATG} / \mathrm{T}$ & ATG/TAA \\
\hline $\operatorname{trn} G$ & J & $5488-5554$ & $5488-5554$ & 67 & 67 & 0 & -8 & & \\
\hline nad3 & J & $5555-5908$ & $5555-5908$ & 354 & 354 & 0 & 0 & ATA/TAA & ATA/TAA \\
\hline $\operatorname{trn} R$ & J & $5907-5970$ & $5907-5970$ & 64 & 64 & -2 & -2 & & \\
\hline $\operatorname{trn} A$ & J & $5974-6038$ & $5971-6036$ & 65 & 66 & 3 & 0 & & \\
\hline $\operatorname{trn} N$ & J & 6039-6105 & $6037-6103$ & 67 & 67 & 0 & 0 & & \\
\hline $\operatorname{trnS1}$ & $N$ & $6106-6172$ & $6104-6170$ & 67 & 67 & 0 & 0 & & \\
\hline $\operatorname{trn} E$ & J & $6174-6239$ & $6172-6237$ & 66 & 66 & 1 & 1 & & \\
\hline $\operatorname{trnF}$ & $\mathrm{N}$ & 6238-6304 & $6236-6302$ & 67 & 67 & -2 & -2 & & \\
\hline nad5 & $N$ & 6304-8046 & $6302-8017$ & 1743 & 1766 & -1 & -1 & GTG/TAA & ATT/TAA \\
\hline $\operatorname{trnH}$ & $\mathrm{N}$ & $8047-8110$ & $8045-8109$ & 64 & 65 & 0 & 27 & & \\
\hline nad4 & $N$ & $8111-9452$ & $8113-9451$ & 1342 & 1339 & 0 & 3 & $\mathrm{ATG} / \mathrm{T}$ & ATG/T \\
\hline nad4L & $\mathrm{N}$ & $9446-9745$ & $9445-9744$ & 300 & 300 & -7 & -7 & ATG/TAA & ATG/TAA \\
\hline $\operatorname{trn} T$ & J & $9752-9816$ & $9751-9815$ & 65 & 65 & 6 & 6 & & \\
\hline $\operatorname{trn} P$ & N & $9817-9882$ & $9816-9881$ & 66 & 66 & 0 & 0 & & \\
\hline nad6 & J & $9885-10,409$ & $9884-10,408$ & 525 & 525 & 2 & 2 & ATT/TAA & ATT/TAA \\
\hline$c o b$ & J & $10,409-11,545$ & $10,408-11,544$ & 1137 & 1137 & -1 & -1 & ATG/TAA & ATG/TAA \\
\hline $\operatorname{trnS2}$ & J & $11,544-11,609$ & $11,543-11,608$ & 66 & 66 & -2 & -2 & & \\
\hline nadl & $\mathrm{N}$ & $11,628-12,572$ & $11,629-12,573$ & 945 & 945 & 18 & 20 & ATT/TAA & ATT/TAA \\
\hline $\operatorname{trn} L 1$ & N & $12,579-12,644$ & $12,580-12,645$ & 66 & 66 & 6 & 6 & & \\
\hline$r r n L$ & $\mathrm{~N}$ & $12,645-13,972$ & $12,646-13,973$ & 1328 & 1328 & 0 & 0 & & \\
\hline $\operatorname{trnV}$ & $\mathrm{N}$ & $13,973-14,044$ & $13,974-14,044$ & 72 & 72 & 0 & 0 & & \\
\hline$r r n s$ & $\mathrm{~N}$ & $14,045-14,841$ & $14,045-14,838$ & 797 & 794 & 0 & 0 & & \\
\hline$C R$ & & $14,842-15,416$ & $14,839-15,418$ & 575 & 579 & 0 & 0 & & \\
\hline
\end{tabular}

based on 163 morphological characters for 64 species in the subfamily Anophelinae using the approximate weighting (AW) method showed that the subgenera Lophopodomyia, Stethomyia, Kerteszia, Nyssorhynchus and Cellia were monophyletic, whereas the subgenus Anopheles was polyphyletic. Two subgenera, Lophopodomyia and Stethomyia, were nested within the subgenus Anopheles [12]. A later morphology-based phylogenetic analysis, which used 167 characters for 66 species in the Anophelinae analyzed with both the equal weighting (EW) and implied weighting (IW) methods, found the same results as described above [14]. All analyses from these three methods showed that the subgenera Nyssorhynchus and Kerteszia were sister-taxa, while the AW and EW methods suggested that the Nyssorhynchus + Kerteszia was sister-group to subgenus 


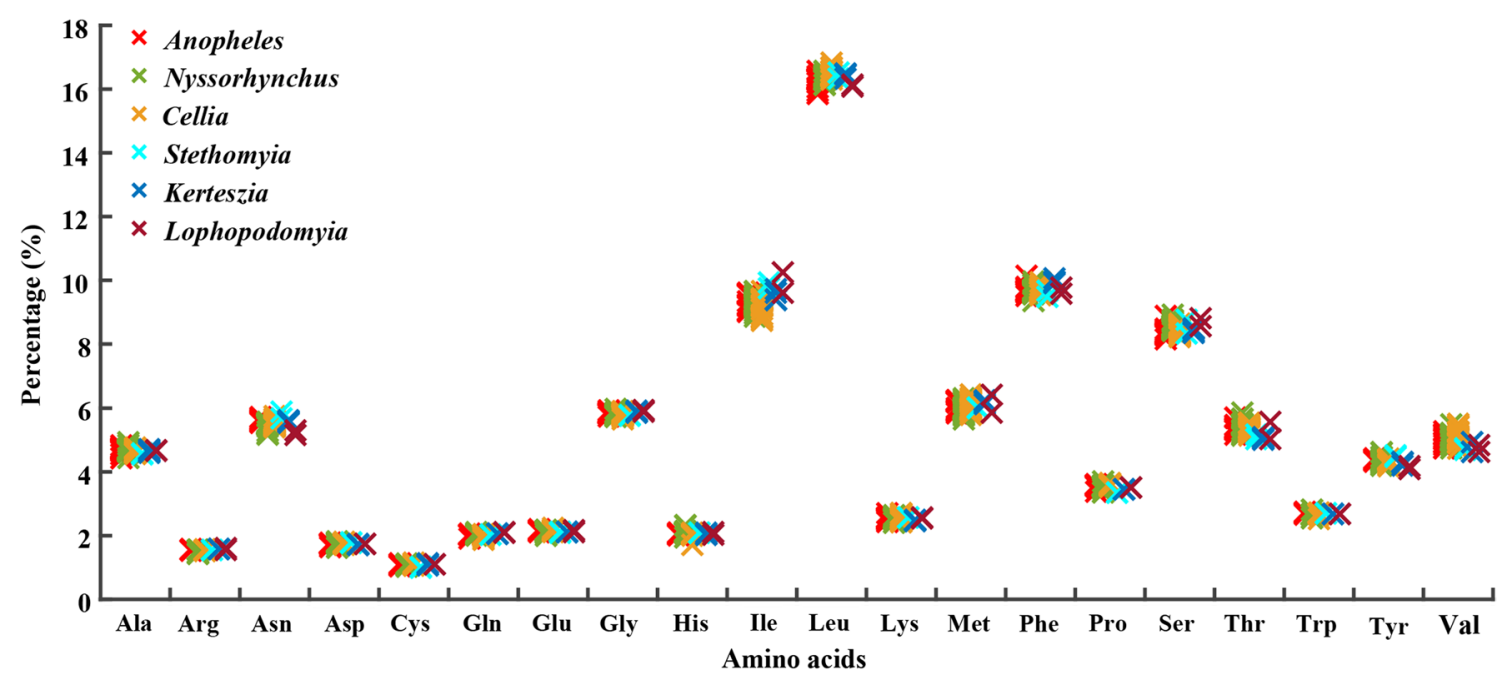

Fig. 3 Frequency percentage of each of 20 coded amino acids in 76 mitochondrial genome sequences of the genus Anopheles

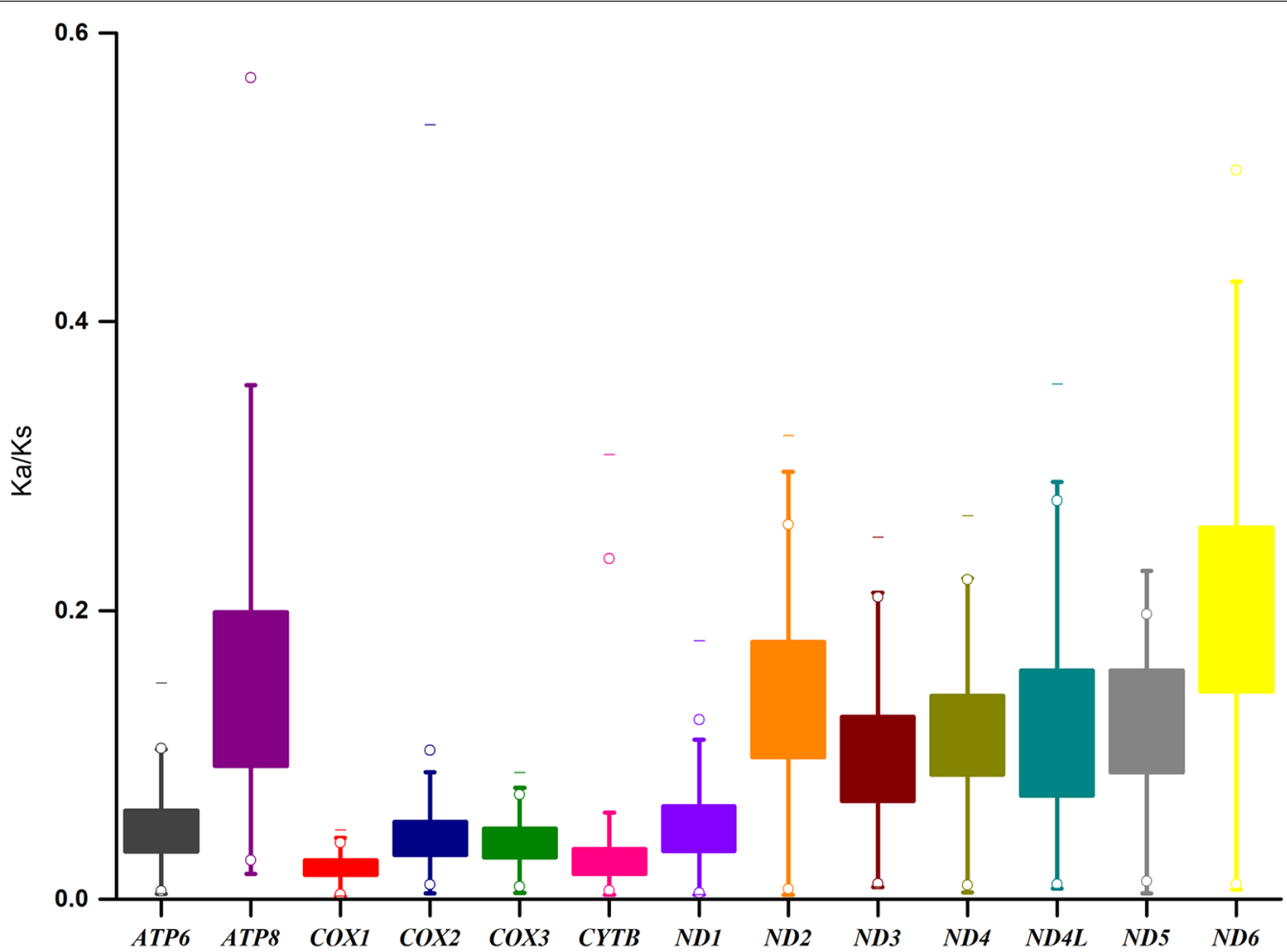

Fig. 4 Evolutionary rates of 13 protein-coding genes (PCGs) within 76 mitochondrial genomes of the genus Anopheles. Ka: Non-synonymous mutation rate; Ks: Synonymous mutation rate; Ka/Ks: The ratio of non-synonymous mutation rate to synonymous mutation rate. Neutral evolution $(\mathrm{Ka} / \mathrm{Ks}=1)$, Purify selection $(\mathrm{Ka} / \mathrm{Ks}<1)$, Positive selection (Ka/Ks $>1)$

Cellia + subgenera Lophopodomyia, Stethomyia and Anopheles, and the IW method found a clade comprising the sister-taxon (Nyssorhynchus + Kerteszia) and subgenus Cellia, and the this clade was sister-group to three subgenera Lophopodomyia, Stethomyia and Anopheles. In contrast, a molecular-based phylogenetic analysis, using COI, COII and 5.8S rRNA for 47 species of Anopheles and using the ML method, supported the monophyly 


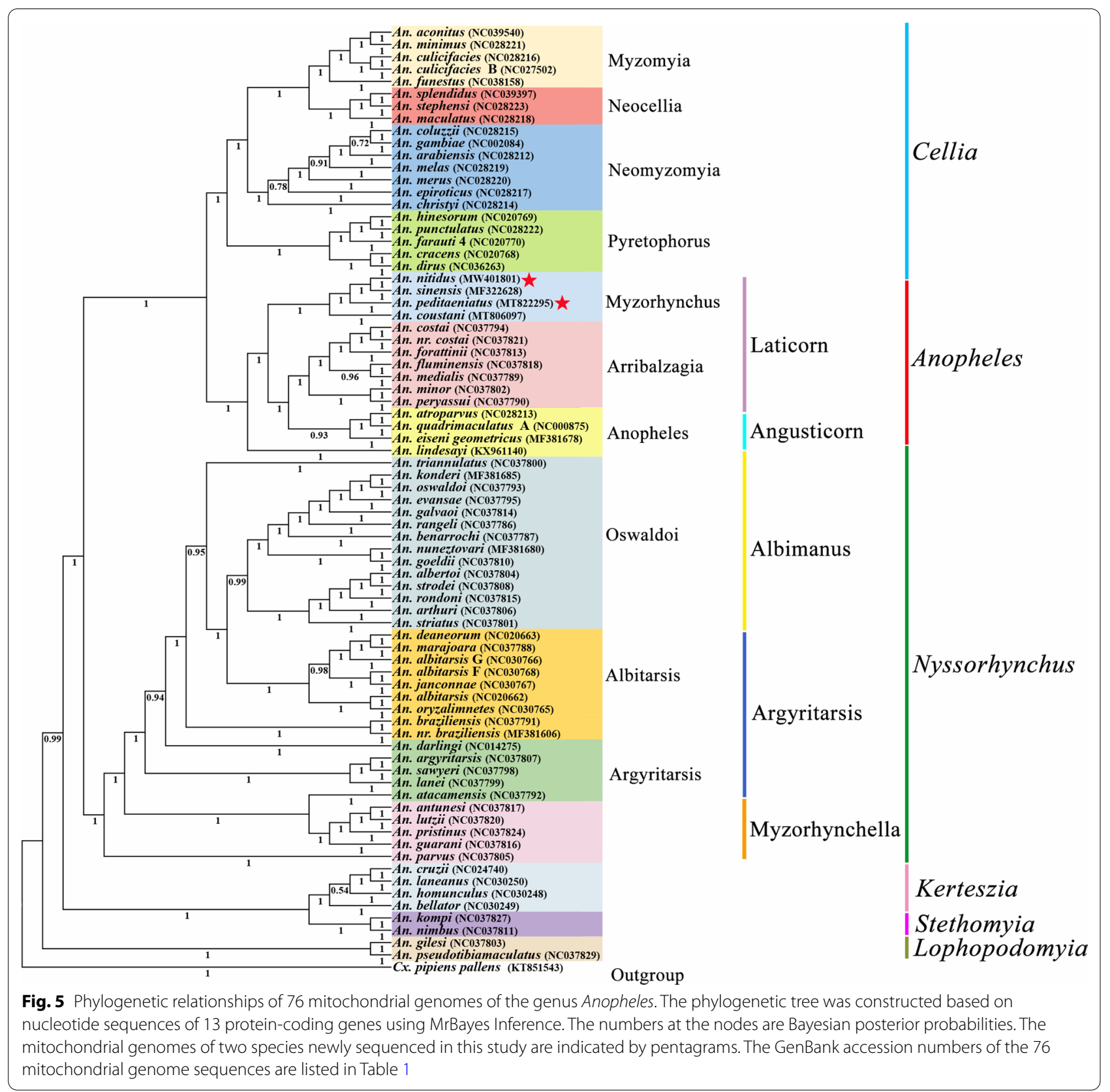

of the subgenera Stethomyia, Kerteszia, Nyssorhynchus, Anopheles and Cellia, and this study suggested the subgenus Anopheles was sister-group to all other subgenera, and placed the subgenus Cellia as a sister-group to a clade which comprised subgenus Nyssorhynchus and a sister-taxon (Stethomyia + Kerteszia) [48]. A recent study of amino acid sequences of 1085 single-copy orthologous genes from 18 species in the subgenera Nyssorhynchus, Anopheles and Cellia analyzed with the ML method found that all three subgenera were monophyletic, and showed that the subgenus Nyssorhynchus was sister to a sister-taxon (Anopheles + Cellia) [49]. Our prior study using mitogenome PCG nucleotide sequences from 50 species in Culicidae with the ML and BI methods showed that the subgenera Nyssorhynchus, Anopheles and Cellia were monophyletic, with the sister relationship between subgenus Nyssorhynchus and a sister-taxon (Anopheles + Cellia) [16].

All six Anopheles subgenera included in the comprehensive phylogenetic analyses discussed above were suggested to be monophyletic except for the subgenus Anopheles, which was recognized as polyphyletic in both 


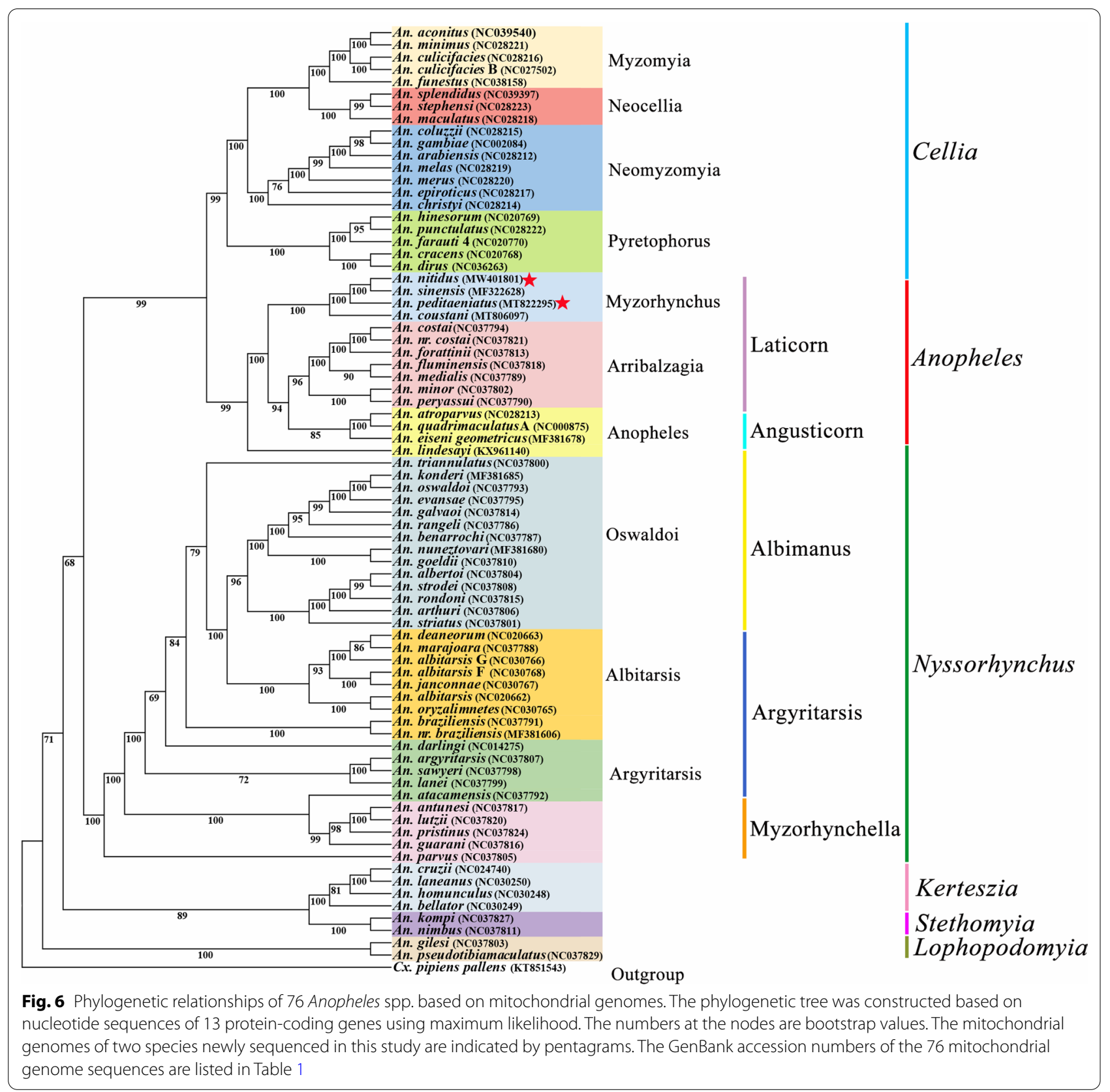

morphology-based inferences, while it was monophyletic in the three molecular-based inferences. Importantly, the study based on 18 whole nuclear genomes showed that the subgenus Anopheles was monophyletic [49]. The present study supported the monophyly of all six subgenera. Studies based on 18 whole nuclear genomes [50] and 50 whole mitogenomes [16] both suggested that the subgenus Nyssorhynchus was sister to the sister-group (Anopheles + Cellia), as does the present study. A recent study based on COI, COII and 5.8S rRNA found that the subgenera Stethomyia and Kerteszia were sisters [48], as in the present study. The subgenus Lophopodomyia was grouped with the subgenera Anopheles and Stethomyia in both morphology-based studies [12, 14], whereas it has not previously been included in molecular-based studies $[16,48,49]$. The current study found that Lophopodomyia was sister to the other five subgenera. In general, the phylogenetic relationships inferred from morphology and those based on molecular data are quite different, and further studies are needed including more species and data to elucidate relationships among subgenera. 
Within the subgenus Cellia, the four series Neomyzomyia, Pyretophorus, Neocellia and Myzomyia that were investigated all appear to be monophyletic ( $\mathrm{pp}=1$ and bv $=100 \%$ for their clades), and Neomyzomyia was a sister-group to all other three series, and Pyretophorus was a sister to the sister-taxon (Neocellia + Myzomyia). The current results are consistent with those from our earlier study, those also based on whole mitogenomes [16], and almost close to those based on 18S, 28S, COI and COII data in both taxon monophyly and relationships [50]. However, the early morphology-based study found all four series to be paraphyletic [12]. These suggest that results stemmed from molecular and morphology are often conflicting as discussed above.

Within the subgenus Anopheles, the two sections Angusticorn (from which only series Anopheles was included) and Laticorn (two series Myzorhynchus and Arribalzagia included) are both polyphyletic. The series Myzorhynchus and Arribalzagia are both monophyletic ( $\mathrm{pp}=1$ and $\mathrm{bv} \geq 96 \%$ for their clades), while if $A n$. lindesayi were excluded, the series Anopheles would also be monophyletic ( $\mathrm{pp}=0.93$ and $\mathrm{bv}=85 \%)$, with the sister relationship between Anopheles and a sister-taxon (Myzorhynchus + Arribalzagia). Analysis of COI, COII and 5.8S rRNA suggested that the sections Laticorn and Angusticorn and the series Anopheles and Myzorhynchus were polyphyletic. In one morphology-based study, the sections Laticorn and Angusticorn and the series Myzorhynchus and Anopheles were paraphyletic [12]. The other morphology study found section Laticorn and the series Arribalzagia and Myzorhynchus to be monophyletic, while section Angusticorn and the series Anopheles were polyphyletic [14]. All of these studies suggested that section Angusticorn and series Anopheles were polyphyletic, and most studies found the section Laticorn to be polyphyletic, whereas series Arribalzagia was always monophyletic while series Myzorhynchus may be monophyletic.

Within the subgenus Nyssorhynchus, three sections, Myzorhynchella, Argyritarsis and Albimanus, were investigated, and the subdivisions in all three sections all appear to be polyphyletic or paraphyletic. A morphology study suggested that sections Albimanus, Argyritarsis and Myzorhynchella were paraphyletic [12]. Two molecular studies found the three sections to be not monophyletic, [51, 52]. All four studies demonstrate that the taxonomy and phylogenetics of Nyssorhynchus are quite conflicted, with more study necessary to reconstruct their taxonomic system.

\section{Conclusions}

This study analyzed the complete mitogenomes of $A n$. peditaeniatus and An. nitidus and investigated phylogenetic relationships among 76 species in the genus Anopheles. These mitogenomes have the same general characteristics found in earlier reports from insects; however, the $\operatorname{trn} R$ and $\operatorname{trn} A$ are reversed in comparison to other Diptera mitogenomes, as has been reported in other genera in the Culicidae. Genome variations mainly occur in the $\mathrm{CR}$ regions, which range in length from 493 to $886 \mathrm{bp}$ and have six repeat regions, identified for the first time. The subgenera Lophopodomyia, Stethomyia, Kerteszia, Nyssorhynchus, Anopheles and Cellia were all found to be monophyletic and showed a new phylogenetic relationship among the six subgenera investigated. Four series Neomyzomyia, Pyretophorus, Neocellia and Myzomyia in the subgenus Cellia, were found to be monophyletic, as were the series Arribalzagia and Myzorhynchus in the subgenus Anopheles, while the series Anopheles and three sections in Nyssorhynchus, Myzorhynchella, Argyritarsis and Albimanus, and their subdivisions were polyphyletic or paraphyletic. Further studies of more mosquito species are needed to elucidate the phylogenetic relationships in the genus Anopheles.

\section{Abbreviations}

Mitogenome: Mitochondrial genome; PCGs: Protein-coding genes; rRNAs: Ribosomal RNA genes; tRNAs: Transfer RNA genes; CR: Control region; RSCU: Relative synonymous codon usage; BI: Bayesian inference; ML: Maximum likelihood.

\section{Supplementary Information}

The online version contains supplementary material available at https://doi. org/10.1186/s13071-021-04963-4.

Additional file 1: Table S1. Composition and skewness of 76 mitochondrial genomes of the genus Anopheles.

Additional file 2: Table S2. Relative synonymous codon usage (RSCU) in 76 mitochondrial genomes of the genus Anopheles.

Additional file 3: Figure S1. Predicted secondary structures of 22 tRNAs in the mitochondrial genomes of An. peditaeniatus (a), An. nitidus (b).

Additional file 4: Figure S2. Repeat unit types of the CRs in the 74 mitochondrial genomes of the genus Anopheles. The pentagrams denote poly-T stretch, and the location and copy number of other repeat types are shown by colored dots: orange represents the second type; purple represents the third type ([TA(A)] n Stretch); blue represents the fourth type; pink represents the fifth type; green represents the sixth type. Nonrepeat regions are indicated by colored box.

\section{Acknowledgements}

This research was supported by the National Natural Science Foundation of China $(31872262,31672363)$ and National Key Program of Science and Technology Foundation Work of China (2015FY210300). 


\section{Authors' contributions}

$B C$ and JG conceived and designed the study. JG and BC performed the experiments and data analysis, and drafted the manuscript. ZTY, WBF, HY and XDL participated in specimen collection and experiments. All authors read and approved the final manuscript.

\section{Funding}

This research was supported by the National Natural Science Foundation of China $(31872262,31672363)$ and National Key Program of Science and Technology Foundation Work of China (2015FY210300).

\section{Availability of data and materials}

All data are available as tables and figures in the main document and its additional files. The GenBank accession numbers for the two mitogenomes produced in the present study are MW401801 and MT822295.

\section{Declarations}

Ethics approval and consent to participate

Not applicable.

\section{Consent for publication}

Not applicable.

\section{Competing interests}

The authors declare that they have no competing interests.

Received: 28 April 2021 Accepted: 17 August 2021

Published online: 06 September 2021

\section{References}

1. Harbach RE. An Anopheles by any other name...? J Med Entomol. 2018;55:1069-70.

2. WHO. World Malaria Report 2020. Geneva: World Health Organization; 2020.

3. Derua YA, Alifrangis M, Magesa SM, Kisinza WN, Simonsen PE. Sibling species of the Anopheles funestus group, and their infection with malaria and lymphatic filarial parasites, in archived and newly collected specimens from northeastern Tanzania. Malaria J. 2015;14:104.

4. Sim C, Hong YS, Vanlandingham DL, Harker BW, Christophides GK, Christophides FC, et al. Modulation of Anopheles gambiae gene expression in response to o'nyong-nyong virus infection. Insect Mol Biol. 2005;14:475-81

5. Theobald FV. The classification of the Anophelina. J Trop Med. 1902;5:181-3.

6. Christophers SR. The male genitalia of Anopheles. Indian J Med Sci. 1915:3:371-94.

7. Edwards FW. Genera Insectorum. Diptera, Fam. Culicidae. Fascicle 194 Bruxelles. Belgium: Desmet Verteneuil; 1932.

8. Komp WHW. The species of the subgenus Kerteszia of Anopheles (Diptera, Culicidae). Ann Entomol Soc Am. 1937;30:492-529.

9. Antunes PCA. A new Anopheles and a new Goeldia from Colombia (Dipt. Culic.). Bull Entomol Res. 1937:28:69-73.

10. Harbach RE, Rattanarithikul R, Harrision BA. Baimaia, a new subgenus for Anopheles kyondawensis Abraham, a unique crabhole-breeding anopheline in Southeastern Asia. P Entomol Soc Wash. 2005;107:750-61.

11. Harbach RE, Kitching IJ. The phylogeny of Anophelinae revisited: inferences about the origin and classification of Anopheles (Diptera: Culicidae). Zool Scr. 2015:45:34-47.

12. Sallum MAM, Schultz TR, Wilkerson RC. Phylogeny of Anophelinae (Diptera Culicidae) based on morphological characters. Ann Entomol Soc Am. 2000:93:745-75

13. Collucci E, Sallum MAM. Phylogenetic analysis of the subgenus Kerteszia of Anopheles (Diptera: Culicidae: Anophelinae) based on morphological characters. Insect Syst Evol. 2003;34:361-72.

14. Harbach RE, Kitching IJ. Reconsideration of Anopheline mosquito phylogeny (Diptera: Culicidae: Anophelinae) based on morphological data. Syst Biodivers. 2005:3:345-74.
15. Fateh K, Ali OM, Mahdi SM, Waterhouse RM, Hasan V Ali HBA et al. Phylogenetic analysis of the Oriental Palearctic Afrotropical members of Anopheles (Culicidae: Diptera) based on nuclear rDNA and mitochondrial DNA characteristics. Jpn J Infect Dis. 2014;67:361-7.

16. Hao YJ, Zou YL, Ding YR, Xu WY, Yan ZT, Li XD, et al. Complete mitochondrial genomes of Anopheles stephensi and Anopheles dirus and comparative evolutionary mitochondriomics of 50 mosquitoes. Sci Rep. 2017;7:7666.

17. Mao QM, Li TJ, Fu WB, Yan ZT, Chen B. Sequencing of the complete mitochondrial genome of Anopheles lindesayi and a phylogenetic analysis of the genus Anopheles (Diptera: Culicidae) based on mitochondrial genomes. Acta Entomol Sinica. 2019;62:103-18.

18. Jl B. Animal mitochondrial genomes. Nucleic acids res. 1999;27:1767-80.

19. Shao R, Barker SC. Mitochondrial genomes of parasitic arthropods: implications for studies of population genetics and evolution. Parasitology. 2007:134:153-67.

20. Cameron SL. Insect mitochondrial genomics: implications for evolution and phylogeny. Annu Rev Entomol. 2013:59:95-117.

21. Clary DO, Wolstenholme DR. The mitochondrial DNA molecular of Drosophila yakuba: nucleotide sequence, gene organization, and genetic code. J Mol Evol. 1985;22:252-71.

22. Cameron SL, Lambkin CL, Barker SC, Whiting MF. A mitochondrial genome phylogeny of Diptera: whole genome sequence data accurately resolve relationships over broad timescales with high precision. Syst Entomol. 2007:32:40-59.

23. Chang HH, Qiu ZY, Yuan H, Wang XY, Li XJ, Sun HM, et al. Evolutionary rates of and selective constraints on the mitochondrial genomes of Orthoptera insects with different wing types. Mol Phylogenet Evol. 2020;145:106734.

24. Yuan ML, Zhang QL, Zhang L, Guo ZL, Liu YJ, Shen YY, et al. High-leve phylogeny of the Coleoptera inferred with mitochondrial genome sequences. Mol Phylogenet Evol. 2016;104:99-111.

25. Tang P, Zhu JC, Zheng BY, Wei SJ, Sharkey M, Chen XX, et al. Mitochondrial phylogenomics of the Hymenoptera. Mol Phylogenet Evol. 2018;131:8-18

26. Zhang NX, Yu G, Li TJ, He QY, Zhou Y, Si FL, et al. The complete mitochondrial genome of Delia antiqua and its implications in dipteran phylogenetics. PLOS ONE. 2015:10:e0139736.

27. Lu BL. Fauna Sinica. Insecta. Diptera: Culicidae 1, vol. 8. Beijing: Science Press; 1997.

28. Yang FL, Li XD, Yan ZT, Chen B. The molecular identification markers of Anopheles sinensis. Chongqing Normal Univ (Nat Sci). 2014:31:40-4.

29. Patel RK, Mukesh J, Liu Z. NGS QC Toolkit: a toolkit for quality control of next generation sequencing data. PLoS ONE. 2012;7:e30619.

30. Bankevich A, Nurk S, Antipov D, Gurevich AA, Dvorkin M, Kulikov AS, et al. SPAdes: a new genome assembly algorithm and its applications to single-cell sequencing. J Comput Biol. 2012;19:455-77.

31. Bernt M, Donath A, Juhling F, Externbrink F, Florentz C, Fritzsch $G$, et al. MITOS: improved de novo metazoan mitochondrial genome annotation. Mol Phylogenet Evol. 2013;69:313-9.

32. Kearse M, Moir R, Wilson A, Stones-Havas S, Cheung M, Sturrock S, et al. Geneious basic: an integrated and extendable desktop software platform for the organization and analysis of sequence data. Bioinformatics. 2012;28:1647-9.

33. Lohse M, Drechsel O, Bock R. Organellar Genome DRAW (OGDRAW): a tool for the easy generation of high-quality custom graphical maps of plastid and mitochondrial genomes. Curr Genet. 2007;52:267-74.

34. Tamura K, Stecher G, Peterson D, Filipski A, Kumar S. MEGA6: molecular evolutionary genetics analysis version 6.0. Mol Biol Evol. 2013;30:2725-9.

35. Perna NT, Kocher TD. Patterns of nucleotide composition at four-fold degenerate sites of animal mitogenomes. J Mol Evol. 1995;41:353-8.

36. Mikrajuddin A, Khairurrijal A. A simple method for determining surface porosity based on SEM images using Origin Pro software. Indonesian J Phys. 2009;20:37-41.

37. Julio R, Albert FM, Carlos JSD, Sara GR, Pablo L, Sebastián ERO, et al. DnaSP 6: DNA sequence polymorphism analysis of large data sets. Mol Biol Evol. 2017:34:3299-302.

38. Benson G. Tandem repeats finder: a program to analyze DNA sequences. Nucleic Acids Res. 1999:27:573-80. 
39. Vaidya G, Lohman DJ, Meier R. SequenceMatrix: concatenation software for the fast assembly of multi-gene datasets with character set and codon information. Cladistics. 2011;27:171-80.

40. Lanfear R, Frandsen PB, Wright AM, Senfeld T, Calcott B. PartitionFinder 2: new methods for selecting partitioned models of evolution for molecular and morphological phylogenetic analyses. Mol Biol Evol. 2017;34:772-3.

41. Nguyen L, Schmidt H, Haeseler A, Minh B. IQ-TREE: a fast and effective stochastic algorithm for estimating maximum-likelihood phylogenies. Mol Biol Evol. 2015;32:268-74.

42. Ronquist F, Teslenko M, Mark P, Ayres D, Darling A, Hohna S, Larget B, Liu L, Suchard M, Huelsenbeck J. MrBayes 3.2: efficient Bayesian phylogenetic inference and model choice across a large model space. Syst Biol. 2015;61:539-42

43. Beard CB, Hamm D, Collins FH. The mitogenome of the mosquito Anopheles gambiae: DNA sequence, genome organization, and comparisons with mitochondrial sequences of other insects. Insect Mol Biol. 1993;2:103-24.

44. Wolstenholme DR. Animal mitochondrial DNA: structure and evolution. Int Rev Cytol. 1992;141:173-216.

45. Behura SK, Lobo NF, Haas B, Debruyn B, Lovin DD, Shumway MF, et al. Complete sequences of mitochondria genomes of Aedes aegypti and Culex quinquefasciatus and comparative analysis of mitochondrial DNA fragments inserted in the nuclear genomes. Insect Biochem Molec. 2011:41:770-7.

46. Saito S. Replication origin of mitochondrial DNA in insects. Genetics. 2005;171:1695-705.
47. Sun L, Li TJ, Fu WB, Yan ZT, Si FL, Zhang YJ, et al. The complete mt genomes of Lutzia halifaxia, Lt. fuscanus and Culex pallidothorax (Diptera: Culicidae) and comparative analysis of 16 Culex and Lutzia mt genome sequences. Parasite Vector. 2019;12:368-81.

48. Freitas LA, Russo CAM, Voloch CM, Mutaquiha OCF, Marques LP, Schrago $\mathrm{CG}$, et al. Diversification of the genus Anopheles and a Neotropical clade from the Late Cretaceous. PLoS ONE. 2015;10:e0134462.

49. Neafsey DE, Waterhouse RM, Abai MR, Aganezov SS, Alekseyev MA, Allen JE, et al. Mosquito genomics. Highly evolvable malaria vectors: the genomes of 16 Anopheles mosquitoes. Science. 2015;347:1258522.

50. Sallum MAM, SchultzTR, Foster PG, Aronstein K, Wirtz RA, Wilkerson RC. Phylogeny of Anophelinae (Diptera:Culicidae) based on nuclear ribosomal and mitochondrial DNA sequences. Syst Entomol. 2002;27:361-82.

51. Bourke BP, Foster PG, Bergo ES, Calado DC, Sallum MAM. Phylogenetic relationships among species of Anopheles (Nyssorhynchus) (Diptera, Culicidae) based on nuclear and mitochondrial gene sequences. Acta Trop. 2010;114:88-96.

52. Foster $\mathrm{PG}$, Bergo ES, Bourke $\mathrm{BP}$, et al. Phylogenetic analysis and DNAbased species confirmation in Anopheles (Nyssorhynchus). PLOS ONE. 2013;8:e54063.

\section{Publisher's Note}

Springer Nature remains neutral with regard to jurisdictional claims in published maps and institutional affiliations.
Ready to submit your research? Choose BMC and benefit from:

- fast, convenient online submission

- thorough peer review by experienced researchers in your field

- rapid publication on acceptance

- support for research data, including large and complex data types

- gold Open Access which fosters wider collaboration and increased citations

- maximum visibility for your research: over $100 \mathrm{M}$ website views per year

At BMC, research is always in progress.

Learn more biomedcentral.com/submissions 